\title{
BMJ Open Systematic review and meta-analysis of clinical significance of autoantibodies for idiopathic pulmonary fibrosis
}

\author{
Hiroyuki Kamiya, ${ }^{1}$ Ogee Mer Panlaqui ${ }^{2}$
}

To cite: Kamiya $\mathrm{H}$, Panlaqui $\mathrm{OM}$. Systematic review and metaanalysis of clinical significance of autoantibodies for idiopathic pulmonary fibrosis. BMJ Open 2019;9:e027849. doi:10.1136/ bmjopen-2018-027849

- Prepublication history and additional material for this paper are available online. To view, please visit the journal (http:// dx.doi.org/10.1136/bmjopen2018-027849).

Received 10 November 2018 Revised 26 March 2019 Accepted 27 March 2019

Check for updates

(C) Author(s) (or their employer(s)) 2019. Re-use permitted under CC BY-NC. No commercial re-use. See rights and permissions. Published by BMJ.

${ }^{1}$ Population and Global Health, University of Western Australia, Perth, Western Australia,

Australia

${ }^{2}$ Intensive Care Medicine, Northern Hospital, Melbourne, Victoria, Australia

Correspondence to

Hiroyuki Kamiya;

mlb04194@nifty.com

\section{ABSTRACT}

Objective To clarify clinical significance of the sole presence of autoantibodies for idiopathic pulmonary fibrosis (IPF) without any other symptoms or signs suggestive of autoimmune disease.

Design Systematic review and meta-analysis Data sources Medline, EMBASE, Science Citation Index Expanded and Google Scholar were searched from 1 January 2002 through 12 February 2019.

Eligibility criteria for selecting studies Primary studies addressing all-cause mortality and the development of a defined autoimmune disease for IPF with autoantibodies were included for the review.

Data extraction and synthesis Two reviewers extracted relevant data and assessed risk of bias independently. Meta-analysis was conducted using a random-effects model if three or more studies reported the same outcome for a certain autoantibody. The quality of evidence was assessed by the Grades of Recommendation, Assessment, Development and Evaluation system.

Results Out of 4603 records retrieved nine studies were included in this review. All studies contained some risk of bias. Based on pooled data myeloperoxidase antineutrophil cytoplasmic antibody (MP0-ANCA) was significantly associated with microscopic polyangiitis incidence with risk ratio (RR) of 20.2 (95\% Cl: 7.22 to 56.4) and antinuclear antibody (ANA) was also significantly associated with the development of connective tissue diseases with RR of 7.11 ( $p=0.001$ ) ( 10 cases in 157 patients with ANA) in one study. However, there was no significant association of autoantibodies with all-cause mortality aside from MPO-ANCA and proteinase 3-ANCA in one study each. MPO-ANCA was not demonstrated to be associated with all-cause mortality by meta-analysis. The quality of evidence was deemed as either low or very low. Conclusions The presence of autoantibodies such as MPO-ANCA and ANA was demonstrated to be associated with the development of some autoimmune diseases for patients with IPF although there was no difference of allcause mortality. However, the results should be interpreted with caution due to low evidence level.

PROSPERO registration number CRD42017077336.

\section{INTRODUCTION}

Idiopathic pulmonary fibrosis (IPF) is chronic fibrosing interstitial pneumonia of unknown aetiology and the most common type among idiopathic interstitial pneumonias (IIPs).

\section{Strengths and limitations of this study}

A study design that made it possible to accumulate and present current evidence regarding clinical significance of the sole presence of autoantibodies for idiopathic pulmonary fibrosis, which was previously reported in a small study.

- A difficulty in combining all of the results due to substantial heterogeneity between studies.

- Inconclusive results due to a small number of studies and the scarcity of multivariate analyses.

IPF is characterised by pathological patterns of usual interstitial pneumonia (UIP) and diagnosed based on the exclusion of known causes such as connective tissue diseases (CTDs), drug toxicity and dust exposure. ${ }^{2}$ However, it is often difficult to exclude all of these possible causes, in particular, underlying CTDs. This is because interstitial pneumonia can be the sole presenting manifestation $^{3}$ or CTDs may be unrecognised due to subtle clinical presentations. ${ }^{4}$ In addition, all symptoms or signs suggestive of CTDs may not necessarily emerge at the same time..$^{5}$ As a result, some patients cannot be diagnosed as CTD-IP regardless of an implication of autoimmune features. ${ }^{6}$ Historically, these cases were described using diverse nomenclatures such as undifferentiated CTD-associated $\mathrm{IP}^{7}$ lung-dominant $\mathrm{CTD}^{8}$ and autoimmune-featured interstitial lung disease (ILD). ${ }^{9}$ Recently, a new classification terminology, interstitial pneumonia with autoimmune features (IPAF), has been proposed for research purpose to focus on a more uniform group of patients who can be classified into this possibly distinct clinical entity. ${ }^{10}$ This criteria consist of clinical, serological and morphologic domains. Although UIP is noted to be complicated by some systemic autoimmune disease such as rheumatoid arthritis (RA), ${ }^{11}$ this pathological finding is not a part of classification criteria of IPAF as it is less specific to CTDs 
in contrast to non-specific interstitial pneumonia, which is reported to be the most common pathological change in CTD-IP. ${ }^{12}$ As a result, IPF/UIP with the sole presence of autoantibodies does not meet the IPAF criteria and is still clinically diagnosed as IPF according to the current international guideline. ${ }^{2}$ However, most of the previous reports regarding autoantibodies in patients with IPF were small studies in a single institution ${ }^{13}$ and thus its clinical implications are still uncertain. Therefore, the aim of this systematic review and meta-analysis is to clarify prognostic significance of autoantibodies for IPF. The protocol of this study was registered with PROSPERO (International Prospective Register of Systematic Reviews). ${ }^{14}$

\section{METHODS}

This review was conducted and reported according to the Preferred Reporting Items for Systematic Reviews and Meta-Analyses ${ }^{15}$ and the Meta-analysis of Observational Studies in Epidemiology statement. ${ }^{16}$ The methods of the review were described briefly as the in-depths were previously reported as a protocol paper. ${ }^{17}$

\section{Patient and public involvement}

There was no patient and public involvement in the whole process of conducting this research.

\section{Eligibility}

This review included any type of primary studies aside from a case report where the effect of autoantibodies was comparatively investigated for the outcome of IPF. IPF was diagnosed based on previously published joint statement of American Thoracic Society/European Respiratory Society/Japanese Respiratory Society/Latin American Thoracic Association (ATS/ERS/JRS/ALAT). ${ }^{2}$ If IPF was not mentioned, radiological and/or pathological UIP was also eligible if it was reported as idiopathic excluding possible secondary causes. There was no limitation of follow-up lengths and patients could be included at any point of time during their disease course.

Autoantibodies of interest in this study included both non-specific and specific antibodies against systemic self-antigens such as the ones listed in the serological domain of IPAF, which are closely related to CTDs. ${ }^{10}$ Antineutrophil cytoplasmic antibody (ANCA) targeted against myeloperoxidase (MPO) or proteinase 3 (PR3) also constituted autoantibodies in this study since ANCA-associated vasculitides is reported to be complicated with pulmonary fibrosis, particularly UIP $^{18}$ although this autoantibody was not included in the IPAF classification criteria due to a different disease entity. ${ }^{10}$ The measurement and the reference value of these laboratory tests were determined by each research institution.

Primary outcomes were all-cause and pulmonary-cause mortality. Secondary outcomes were a progression of the disease and a deterioration of health-related quality of life. The development of a defined autoimmune disease over the follow-up periods of time was also included in the secondary outcome.

Only English articles published in 2002 or later were eligible as current classification system of IIPs was established in that year. ${ }^{1}$ Editorials, letters and review articles were all ineligible and conference proceedings were also excluded due to concerns of insufficient information.

\section{Search of studies}

The following electronic databases, Medline (Ovid), EMBASE (Ovid) and Science Citation Index Expanded (Web of Science), were searched using subject headings and text words of study population such as 'idiopathic pulmonary fibrosis' and 'autoantibodies', which were modified depending on each database ${ }^{1920}$ and combined with the sensitive methodology filter for prognosis (from 1 January 2002 through 12 February 2019) (online supplementary e-appendix). Grey literature was sought to be identified through Google Scholar. ${ }^{21}$ Reference lists of eligible studies and relevant review articles were also hand-searched to identify additional reports.

\section{Study selection and data collection}

Two reviewers (HK and OMP) independently examined titles and abstracts of all retrieved articles after removing duplicates and selected eligible studies. Data were also extracted by the same reviewers based on the predefined data extraction form ${ }^{17}$ and a disagreement through all these processes was sought to be resolved through discussion between the reviewers. Extracted data included the first author name, publication year, study location, study design, the number of participants and their demographic features, autoantibodies tested, outcomes, methods for statistical analysis, summary statistics and items associated with risk of bias.

\section{Risk of bias in individual studies}

A risk of bias in individual studies was assessed by the Quality in Prognostic Studies tool. ${ }^{22}$ It consisted of six domains, which was rated as either low, moderate or high and a study with low risk of bias in all domains was designated as a study with low risk of bias.

\section{Statistical analysis}

Binary outcomes were to be summarised as the odds ratio $(\mathrm{OR})$, risk ratio (RR) or hazard ratio (HR). If summary statistics were not obtained directly, they were estimated from available data such as the Kaplan-Meier survival curve and the log rank test ${ }^{23}$ or calculated manually based on absolute numbers in two comparative groups. If the outcomes were continuous, the summary effect was to be presented as the mean difference or the difference of the median value.

The results were pooled if the effect of a certain autoantibody on a predefined outcome for patients with IPF was presented by the same summary statistics in three or more studies and otherwise they were reported qualitatively. Univariate results were sought to be combined whereas multivariate results were presented descriptively 
as the final model with adjusted factors would have been diverse between studies. Meta-analysis was conducted by a random-effects model with the DerSimonian and Laird method $^{24}$ using the statistical software, Review Manager (RevMan) V.5.3. The result was presented with the $95 \%$ confidence interval (CI) with statistical significance set at the $5 \%$ level and the $95 \%$ prediction interval (PI) was also calculated. ${ }^{25}$ Heterogeneity was assessed by the $Q$ statistics and the $\mathrm{I}^{2}$. Statistical significance of the test for heterogeneity was set at the $10 \%$ level and the magnitude was interpreted as not important (0\%-30\%), moderate $(30 \%-50 \%)$, substantial $(50 \%-70 \%)$ and considerable $(70 \%-100 \%) .{ }^{26}$ Subgroup analysis was to be conducted based on potential sources of heterogeneity such as the gender and study location (Asia vs non-Asia). Sensitivity analysis was also to be conducted focusing on studies with low risk of bias alone. Small study bias including publication bias was to be examined graphically by a funnel plot and statistically by Egger's test ${ }^{27}$ if 10 or more studies were available. Statistical significance of the test was set at the $10 \%$ level.

\section{Evaluating the presented evidence}

The Grades of Recommendation, Assessment, Development and Evaluation (GRADE) system was applied to evaluate the quality of evidence identified regarding clinical significance of autoantibodies for IPF. ${ }^{28}$

\section{RESULTS}

\section{Search strategy}

A total of 4603 records were retrieved through a search of four electronic databases. After removing 365 duplicates, 1669 articles of ineligible types and 2520 reports of irrelevant subjects through screening titles and abstracts, the remaining 49 articles were retrieved as fulltexts. Out of these reports, 40 failed to meet eligibility criteria and finally nine articles/studies were included in this review. ${ }^{13}{ }^{29-36}$ No additional reports were identified through a hand-search of references of eligible reports and relevant review articles (figure 1).

\section{Characteristics of included studies}

All nine studies were of retrospective cohort design and included a total of 1950 participants. The majority of patients were men with smoking history, and the mean and median age was around 60 s or 70 s. Four studies ${ }^{132930} 32$ tested both non-specific autoantibodies such as antinuclear antibody (ANA) and rheumatoid factor (RF), and specific autoantibodies. One study ${ }^{31}$ only examined ANA and RF, while the other four studies ${ }^{33-36}$ only investigated MPO- and/or PR3-ANCA (table 1). The reference value for both ANA and RF was diverse between studies. ANA was identified in between $1 \%$ and $56.3 \%$ of patients depending on studies, while RF was positive in between $6 \%$ and $17.6 \%$ of patients. MPO-ANCA was tested in seven studies, ${ }^{13}{ }^{29} 32-36$ which demonstrated positivity as between $0 \%$ and $32.1 \%$, while anticyclic citrullinated peptide

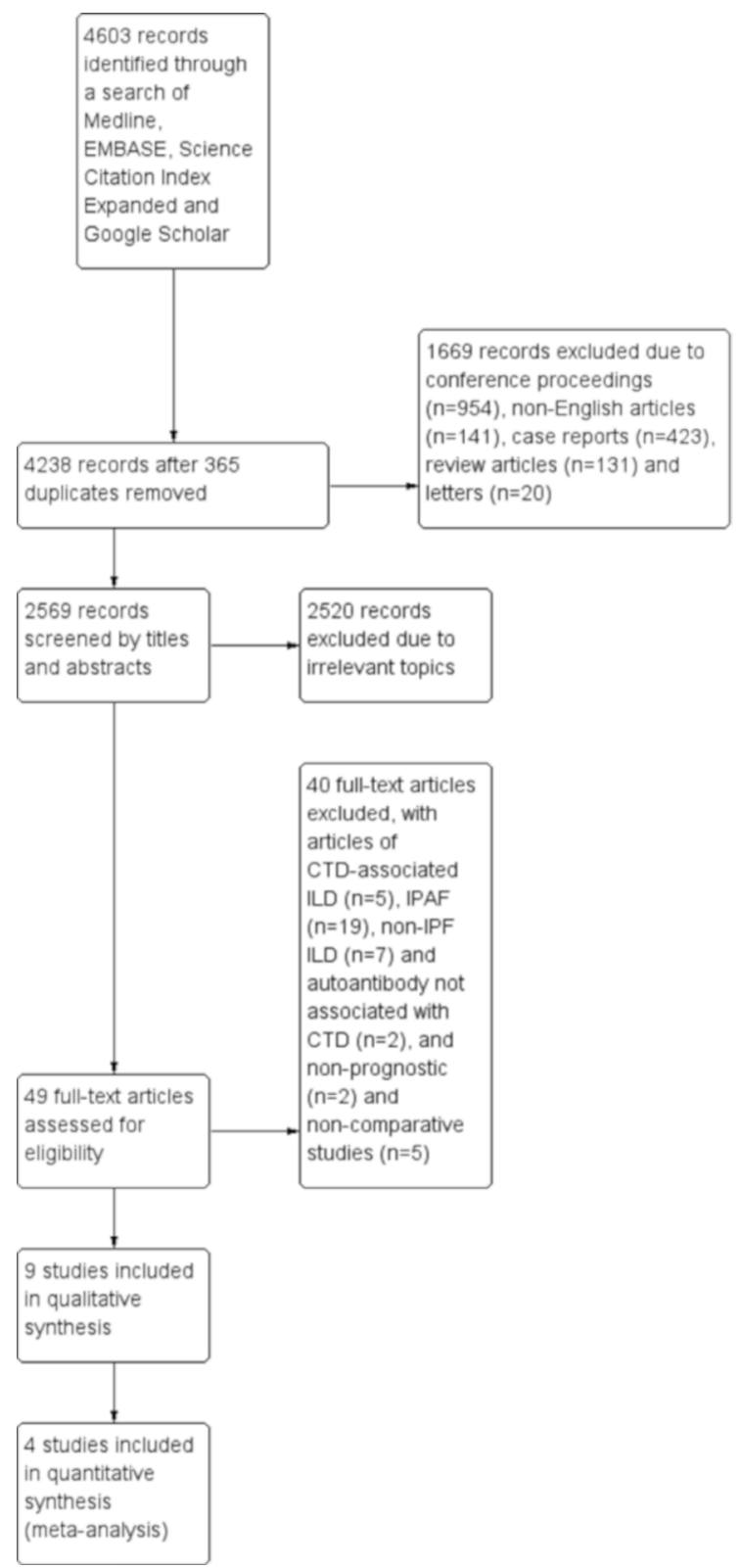

Figure 1 Study flow diagram. A total of 4603 records were retrieved through a search of Medline, EMBASE, Science Citation Index Expanded and Google Scholar. After removing 365 duplicates, 1669 reports were excluded due to ineligible types, which included 954 conference proceedings, 141 nonEnglish articles, 423 case reports, 131 review articles and 20 letters. After excluding 2520 more reports due to irrelevant subjects through screening titles and abstracts, the remaining 49 articles were retrieved as full-texts. Out of these reports, 40 failed to meet eligibility criteria and finally nine articles/ studies were included in this review. Meta-analysis was conducted for four studies. CTD, connective tissue disease; ILD, interstitial lung disease; IPAF, interstitial pneumonia with autoimmune features.

(CCP) antibody was examined in three studies ${ }^{13} 2930$ which reported that it was positive in between $1.0 \%$ and $7.3 \%$. All studies contained some risk of bias, in particular, study attrition was insufficiently explained in all studies. For confounding factors and statistical analysis, all studies 


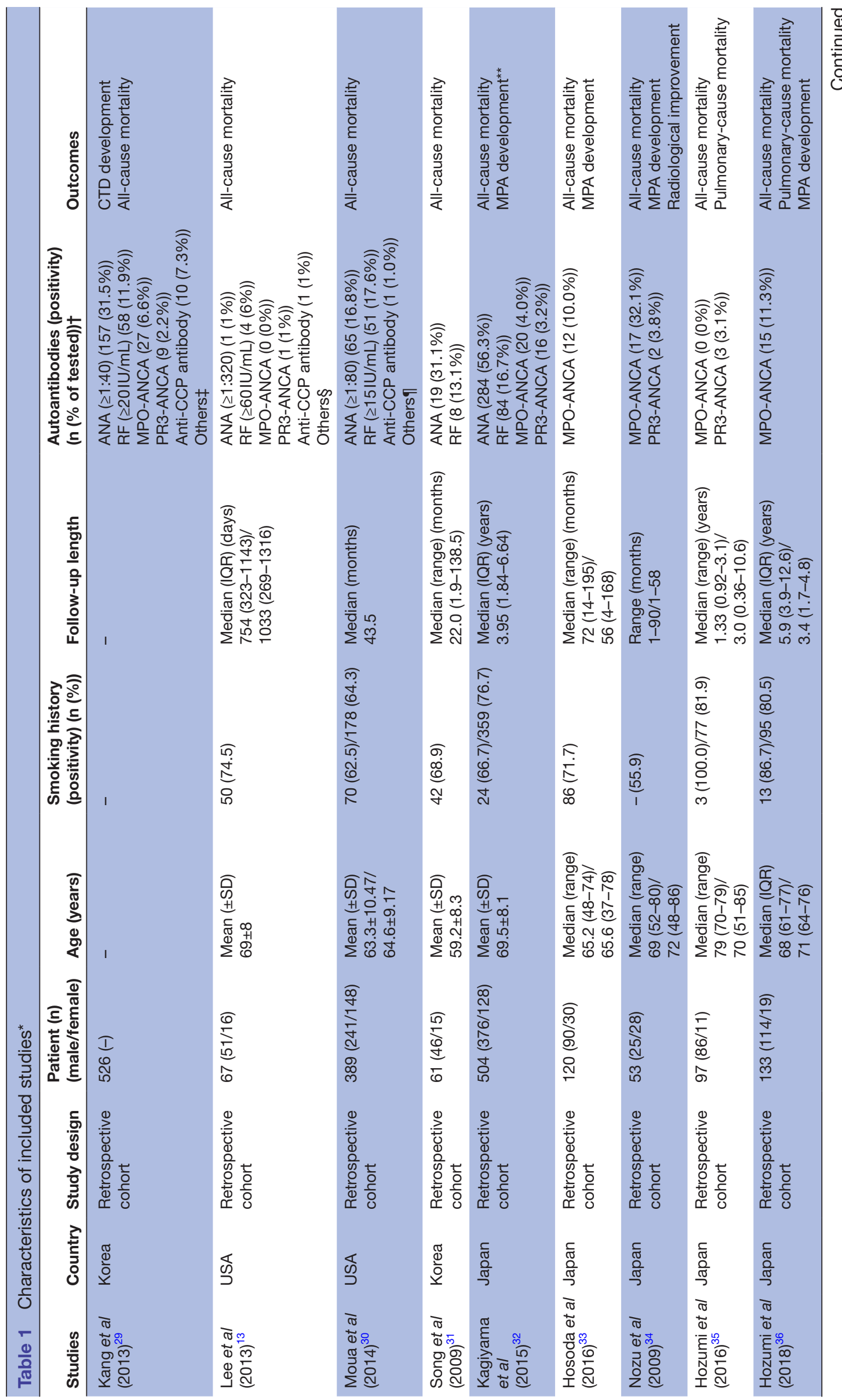


except one had a high risk of bias and one had a medium risk of bias (table 2).

\section{Univariate analysis}

All-cause mortality

Among diverse autoantibodies tested, the effect of ANA and RF on all-cause mortality was reported separately in two studies. Kang et a $l^{29}$ demonstrated no significant difference of the median survival time (MST) between IPF with and without ANA (40.6 vs 46.2 months). Kagiyama $e t a l^{22}$ also reported non-significant result for ANA with HR of 1.31 (95\% CI: 0.98 to 1.76). Kang et a $t^{29}$ demonstrated no significant difference of the MST between IPF with and without RF (44.5 vs 48.8 months). Kagiyama et a ${ }^{32}$ also reported non-significant result for RF with HR of 1.16 (95\%CI: 0.83 to 1.61) (table 3).

The effect of MPO-ANCA on all-cause mortality was reported in three studies. One of these studies demonstrated a significant result with HR of 2.69 (95\% CI: 1.27 to 5.70) (eight deaths in 15 patients with MPO-ANCA), ${ }^{36}$ whereas the results were non-significant and inconsistent in the others with HRs of 1.65 (95\% CI: 0.97 to 2.80$)^{32}$ and 0.58 (95\% CI: 0.25 to 1.38) (five deaths in 12 patients with MPO-ANCA), ${ }^{33}$ respectively. The effect of PR3-ANCA on all-cause mortality was reported in two studies. ${ }^{32} 35$ The former study demonstrated a significant result with HR of 2.99 (95\% CI: 1.52 to 5.86) and the latter study also reported the same trend with RR of 1.79 (95\% CI: 0.77 to 4.16) (two deaths in three patients with PR3-ANCA) although it was not statistically significant. ANCA was not significantly associated with all-cause mortality with HR of 0.60 (95\% CI: 0.23 to 1.57 ) (six deaths in 19 patients with ANCA) in one study. ${ }^{34}$ As approximately $90 \%$ of ANCA were composed of MPO-ANCA in Nozu et al, ${ }^{34}$ the result of the study was combined with those of the other three studies that reported MPO-ANCA. ${ }^{32} 33{ }^{36}$ Meta-analysis of these four studies demonstrated that MPO-ANCA was not significantly associated with all-cause mortality with HR of 1.19 (95\% CI: 0.60 to $2.36 / 95 \%$ PI: 0.06 to 22.2 ) although there was considerable heterogeneity between studies (figure 2).

Three studies, ${ }^{13} 3031$ which reported the effect of the presence of any autoantibody together instead of focusing on a single autoantibody, were excluded from further analysis since such data handling is impractical as it ignores clinical relevance of each autoantibody.

\section{Autoimmune disease incidence}

The development of CTDs was reported in only one study, ${ }^{29}$ which was demonstrated to be $2.5 \%$ of a total of 526 patients. It stated that the most frequently identified CTD was RA $(61.5 \%)$, which was followed by Sjögren syndrome (23.1\%). There was a significant association between the presence of ANA $(\geq 1: 40)$ and CTD incidence with RR of $7.11(p=0.001)$ (10 cases in 157 patients with ANA), whereas RF ( $\geq 20 \mathrm{IU} /$ $\mathrm{mL}$ ) was not significantly associated with CTD incidence with $\mathrm{RR}$ of $2.26(\mathrm{p}=0.19)$ (three cases in 58 patients with RF) (table 3). The development of microscopic polyangiitis (MPA) was reported in four studies ${ }^{32-34} 36$ (table 3). The 
Table 2 Risk of bias in included studies assessed by the Quality in Prognostic Studies tool ${ }^{*}$

\begin{tabular}{lllllll}
\hline Studies & $\begin{array}{l}\text { Study } \\
\text { participation }\end{array}$ & Study attrition & $\begin{array}{l}\text { Prognostic } \\
\text { factor } \\
\text { measurement }\end{array}$ & $\begin{array}{l}\text { Outcome } \\
\text { measurement }\end{array}$ & $\begin{array}{l}\text { Study } \\
\text { confounding }\end{array}$ & $\begin{array}{l}\text { Statistical } \\
\text { analysis and } \\
\text { reporting }\end{array}$ \\
\hline Kang et al $(2013)^{29}$ & Medium risk & High risk & Medium risk & Low risk & High risk & High risk \\
\hline Lee et al $(2013)^{13}$ & Medium risk & High risk & Low risk & Low risk & High risk & Medium risk \\
\hline Moua et al $(2014)^{30}$ & Low risk & High risk & Medium risk & Low risk & Low risk & High risk \\
\hline Song et al $(2009)^{31}$ & High risk & High risk & High risk & Low risk & High risk & High risk \\
\hline Kagiyama et al (2015) $)^{32}$ & Medium risk & High risk & Low risk & Low risk & High risk & High risk \\
\hline Hosoda et al $(2016)^{33}$ & Low risk & High risk & Low risk & Low risk & Medium risk & High risk \\
\hline Nozu et al $(2009)^{34}$ & High risk & High risk & Low risk & Low risk & Medium risk & High risk \\
\hline Hozumi et al $(2016)^{35}$ & Medium risk & High risk & Low risk & Low risk & Medium risk & High risk \\
\hline Hozumi et al $(2018)^{36}$ & High risk & High risk & Medium risk & Low risk & Medium risk & High risk \\
\hline
\end{tabular}

${ }^{*}$ Text in bold referring to high risk of bias.

Table 3 Summary of the effect of autoantibodies for idiopathic pulmonary fibrosis ${ }^{*}$

\section{Outcomes/autoantibodies}

\begin{tabular}{|c|c|c|c|}
\hline $\begin{array}{l}\text { Outcomes/autoantibodies } \\
\text { combination }\end{array}$ & Studies & Univariate $(95 \% \mathrm{Cl})$ & Multivariate $(95 \% \mathrm{Cl})$ \\
\hline \multicolumn{4}{|l|}{ All-cause mortality } \\
\hline ANA $(\geq 1: 40)$ & Kang et al $(2013)^{29}$ & $\begin{array}{l}\text { Median survival time } \\
40.6 \text { vs } 46.2 \text { months }(p=0.72)\end{array}$ & - \\
\hline (Reference value not specified) & Kagiyama et al (2015) $)^{32}$ & HR 1.31 (0.98 to 1.76$)$ & - \\
\hline $\mathrm{RF}(\geq 20 \mathrm{IU} / \mathrm{mL})$ & Kang et al (2013) $)^{29}$ & $\begin{array}{l}\text { Median survival time } \\
44.5 \text { vs } 48.8 \text { months }(p=0.43)\end{array}$ & - \\
\hline (Reference value not specified) & Kagiyama et al (2015) ${ }^{32}$ & HR 1.16 (0.83 to 1.61$)$ & - \\
\hline \multirow[t]{3}{*}{ MPO-ANCA } & Kagiyama et al (2015) $)^{32}$ & HR 1.65 (0.97 to 2.80$)$ & HR 1.48 (0.84 to 2.62 ) \\
\hline & Hosoda et al (2016) & HR 0.58 (0.25 to 1.38$)$ & - \\
\hline & Hozumi et al (2018) ${ }^{36}$ & HR 2.69 (1.27 to 5.70$)$ & - \\
\hline \multirow[t]{2}{*}{ PR3-ANCA } & Kagiyama et al (2015) $)^{32}$ & HR 2.99 (1.52 to 5.86$)$ & HR 2.42 (1.23 to 4.76$)$ \\
\hline & Hozumi et al (2016) ${ }^{35}$ & RR 1.79 (0.77 to 4.16$)$ & - \\
\hline ANCA & Nozu et al (2009) $)^{34}$ & HR $0.60(0.23$ to 1.57$)$ & - \\
\hline \multicolumn{4}{|l|}{ Pulmonary-cause mortality } \\
\hline MPO-ANCA & Hozumi et al (2018) ${ }^{36}$ & RR 1.23 (0.74 to 2.07 ) & - \\
\hline PR3-ANCA & Hozumi et al (2016) ${ }^{35}$ & RR 2.24 (0.95 to 5.28$)$ & - \\
\hline \multicolumn{4}{|l|}{ CTD incidence } \\
\hline ANA $(\geq 1: 40)$ & Kang et al $(2013)^{29}$ & RR $7.11(p=0.001)$ & - \\
\hline $\mathrm{RF}(\geq 20 \mathrm{IU} / \mathrm{mL})$ & Kang et al $(2013)^{29}$ & RR $2.26(p=0.19)$ & - \\
\hline \multicolumn{4}{|l|}{ MPA incidence } \\
\hline \multirow[t]{4}{*}{ MPO-ANCA } & Kagiyama et al (2015) $)^{32}$ & RR 11.7 (3.15 to 43.5$)$ & - \\
\hline & Hosoda et al (2016) ${ }^{33}$ & RR 61.0 (3.35 to 1108.5 ) & - \\
\hline & Nozu et al $(2009)^{34}$ & RR 17.8 (1.02 to 311.6$)$ & - \\
\hline & Hozumi et al (2018) ${ }^{36}$ & RR 99.4 (5.88 to 1679.7$)$ & - \\
\hline \multicolumn{4}{|l|}{ Radiological improvement } \\
\hline ANCA & Nozu et al $(2009)^{34}$ & RR 1.53 (0.99 to 2.36$)$ & - \\
\hline
\end{tabular}




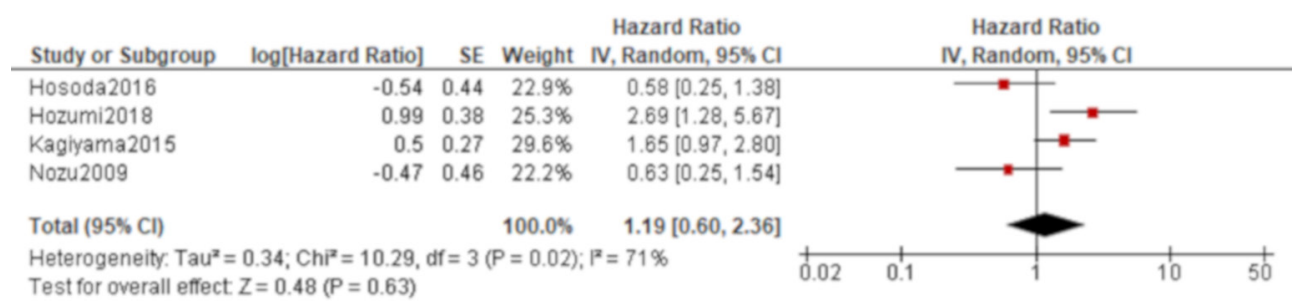

Figure 2 Forest plot of the result of univariate analysis of myeloperoxidase antineutrophil cytoplasmic antibody (MPO-ANCA) for all-cause mortality. The univariate results of MPO-ANCA for all-cause mortality in four studies were pooled and a total of 810 patients were included. MPO-ANCA was not significantly associated with all-cause mortality with $\mathrm{HR}$ of 1.19 (95\% Cl: 0.60 to $2.36, p=0.63)$. The $95 \%$ prediction interval was between 0.06 and 22.2 . There was considerable heterogeneity $\left(X^{2}=10.29\right.$, $\left.\mathrm{p}=0.02, \mathrm{I}^{2}=71 \%\right)$.

incidence of MPA cases was 3, 3, 4 and 6 in 20, 12, 19 and 15 patients with MPO-ANCA in respective studies. Meta-analysis of the results demonstrated higher incidence of MPA with the presence of MPO-ANCA with RR of 20.2 (95\% CI: 7.22 to $56.4 / 95 \%$ PI: 2.11 to 192.8 ) (figure 3).

\section{Other outcomes}

Pulmonary-cause mortality for MPO-ANCA and PR3-ANCA was reported in one study each. Hozumi et al ${ }^{36}$ and Hozumi et al ${ }^{35}$ demonstrated non-significant results with RRs of 1.23 (95\% CI: 0.74 to 2.07) (eight cases in 15 patients with MPO-ANCA) and 2.24 (95\% CI: 0.95 to 5.28) (two cases in three patients with PR3-ANCA), respectively (table 3). Radiological improvement after the corticosteroid and cyclophosphamide therapy was compared between ANCA-positive and negative groups in one study, ${ }^{34}$ which demonstrated non-significant result with RR of 1.53 (95\% CI: 0.99 to 2.36 ) (table 3 ).

\section{Multivariate analysis}

The effect of MPO-ANCA and PR3-ANCA on all-cause mortality was reported using multivariate models in only one study. ${ }^{32}$ MPO-ANCA was not significantly associated with all-cause mortality with HR of 1.48 (95\% CI: 0.84 to 2.62), whereas there was a significant association of PR3-ANCA with all-cause mortality with HR of 2.42 (95\% CI: 1.23 to 4.76) (table 3).

Additional analysis

Neither subgroup analysis nor sensitivity analysis was conducted due to a small number of studies and some risk of bias identified in all studies. Small study bias was unable to be examined due to a small number of studies.

\section{Quality of evidence}

The GRADE system rated the quality of evidence as either low or very low for all of the autoantibodies for IPF (table 4).

\section{DISCUSSION}

This systematic review demonstrated that ANA was the most frequently identified autoantibody for patients with IPF. Based on the result of individual studies, MPO-ANCA and ANA were associated with the development of some autoimmune diseases. However, all-cause mortality was the same regardless of the presence of autoantibodies although MPO-ANCA and PR3-ANCA were demonstrated to be associated with worse prognosis in one study each. Based on the result of pooled data, the presence of MPO-ANCA was significantly associated with the development of MPA although there was no significant difference of all-cause mortality between IPF with and without this autoantibody.

Clinical significance of the sole presence of autoantibodies in IPF has yet to be elucidated. However, it may be related to the pathogenesis of IPF as autoimmunity has been reported to be involved in the development of IPF. ${ }^{37}$ It was demonstrated that CD4 T cells would be activated by some autoantigen inside the lung and help B cells proliferate and produce autoantibodies. ${ }^{39}$ Fibrosis and inflammation will be facilitated by a variety of molecules secreted during this entire process and the

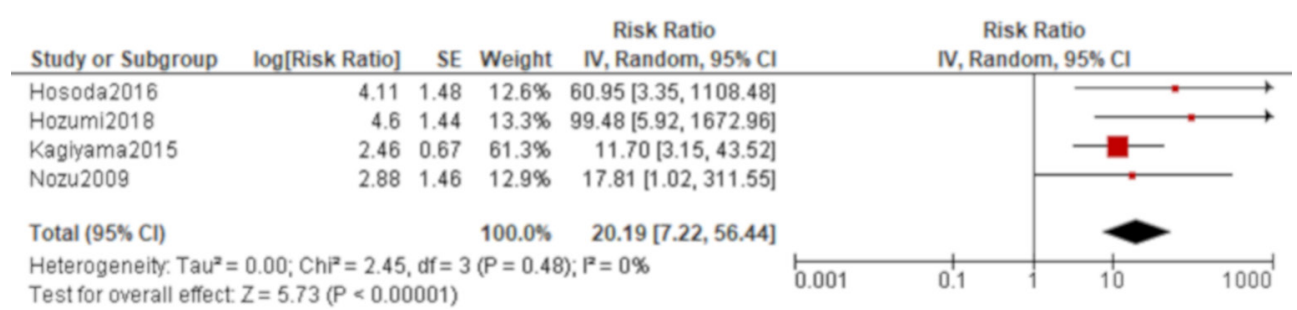

Figure 3 Forest plot of the result of univariate analysis of myeloperoxidase antineutrophil cytoplasmic antibody (MPO-ANCA) for the development of microscopic polyangiitis (MPA). The univariate results of MPO-ANCA for the development of MPA in four studies were pooled and a total of 794 patients were included. MPO-ANCA was significantly associated with the development of MPA with risk ratio (RR) of 20.2 (95\% Cl: 7.22 to $56.4, \mathrm{p}<0.00001)$. The $95 \%$ prediction interval was between 2.11 and 192.8 . There was no heterogeneity $\left(X^{2}=2.45, p=0.48, I^{2}=0 \%\right)$. 


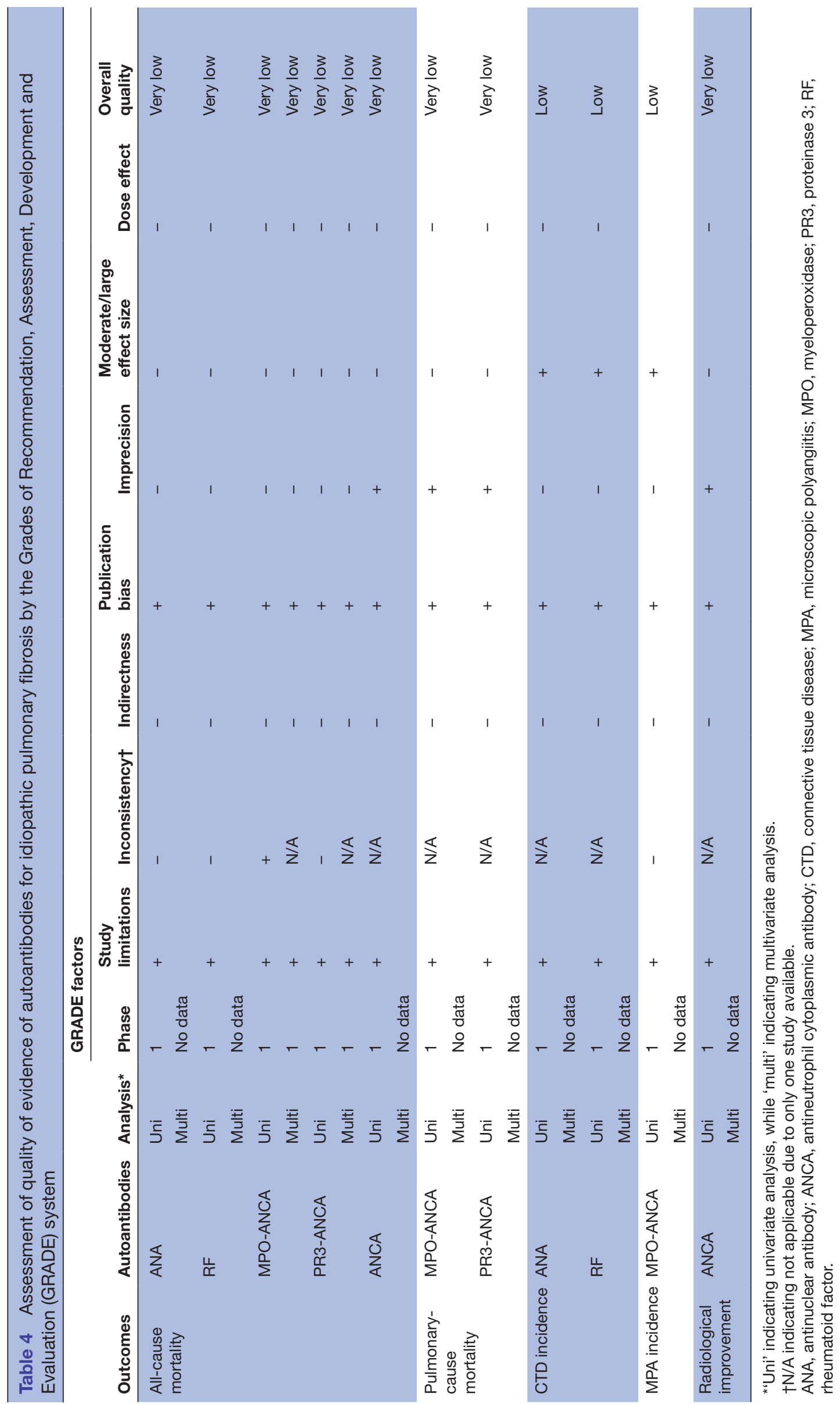

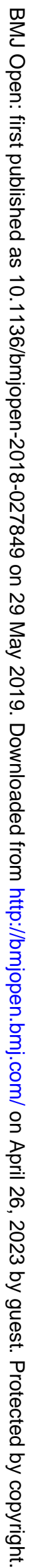


development of IPF may ensue. ${ }^{39}$ Based on this explanation regarding the development of the disease, the presence of autoantibodies in IPF may be reflecting the underlying autoimmunity involved in its pathogenesis rather than suggesting a complication of autoimmune disease. ${ }^{40}$ Another explanation for the presence of autoantibodies in IPF is a chance phenomenon as autoantibodies are noted to be detected in healthy people without any symptoms or signs suggestive of autoimmune disease. ${ }^{41}$ These findings may suggest that autoantibodies in IPF would add no clinical significance and this argument seems to be more likely in a case of non-specific autoantibodies such as ANA and RF. Indeed, the positivity of $\mathrm{RF}$ was between $6 \%$ and $18 \%$ in this study, which is within the normal range for healthy population. ${ }^{42} \mathrm{~A}$ high positive rate of ANA confirmed in this review may also be explained by the fact that all included studies were composed of elderly patients because it is reported that ANA positivity rises as the age increases. ${ }^{41}$ However, the interpretation of non-specific autoantibodies in IPF will be changed depending on their titres. If the titre is high, it may still imply clinical relevance beyond a chance. ${ }^{43} \mathrm{On}$ the other hand, specific autoantibodies such as ANCA and anti-CCP antibody are closely related to a defined autoimmune disease as they directly involve the pathogenesis of the disease. ${ }^{44} 45$ As a result, the presence of specific autoantibodies is likely to predict future development of autoimmune disease. ${ }^{43}$ Although ANA $(\geq 1: 40)$ was demonstrated to be significantly associated with a higher incidence of CTDs in one study in this review, this relationship may have been confounded by the presence of anti-CCP antibody since this autoantibody was identified in $7.3 \%$ of patients and the majority of CTD cases that developed during follow-up periods were RA $(61.5 \%)$. Furthermore, pooled data in this review also demonstrated that MPO-ANCA was significantly associated with the development of MPA. These findings are consistent with previous reports that the majority of UIP preceding CTD cases were manifested as RA and MPA over time. ${ }^{46}$ As ANA is noted to be a less sensitive and specific marker for both of these diseases, ${ }^{43}$ serological testing such as anti-CCP antibody and MPO-ANCA needs to be implemented to avoid a misdiagnosis of IPF and not to miss the chance of a proper treatment.

Although some autoantibodies in IPF were demonstrated to be predictive of future occurrence of autoimmune disease in this review, all autoantibodies aside from PR3-ANCA were not associated with all-cause mortality. There was also no significant difference of all-cause mortality between IPF with and without MPO-ANCA based on pooled data although there was considerable heterogeneity of the effect between studies. The prognosis of ILD is reported to be closely related to both radiological and pathological patterns and its underlying causes. ${ }^{47-49}$ Previous studies mentioned that CTD/UIP usually demonstrates better survival than IPF $/ \mathrm{UIP}^{50}{ }^{50}$ while the prognosis of RA/UIP and MPA/UIP would be similar to that of IPF/UIP. ${ }^{11}{ }^{51}$ Based on these previous reports, the prognosis of IPF with autoantibodies may be different depending on the rate of autoimmune disease incidence and the proportion of underlying RA and MPA cases. In short, if autoantibodies in IPF are all non-specific with low titres, the prognosis may be similar to IPF without autoantibodies as they are likely to confer little additional clinical significance. However, if autoantibodies in IPF are specific or shown positive with high titres even if they are non-specific, the prognosis may depend on possible underlying autoimmune diseases. In the latter case, if IPF with autoantibodies includes a large number of cases that will evolve into RA or MPA, its prognosis may be the same as IPF without autoantibodies, which may be the case demonstrated in this review. Clinical significance of PR3-ANCA in IPF has yet to be elucidated. However, worse prognosis with this autoantibody in this review may be related to a missed diagnosis because it is fairly specific for granulomatosis with polyangiitis, a life-threatening systemic vasculitis. ${ }^{52}$ Another potential factor that can influence clinical course of the disease is treatment. As IPF with autoantibodies is diagnosed as IPF under current diagnostic criteria, ${ }^{2}$ therapeutic agents such as corticosteroids and immunosuppressive drugs may be reserved until it is revealed as a defined autoimmune disease. However, this clinical decision may deprive the chance of improving clinical course of the disease. In particular, IPF with MPO-ANCA may be complicated with acute respiratory failure due to diffuse alveolar haemorrhage caused by capillaritis, which is a critical condition and difficult to be treated ${ }^{53}$ An early intervention for IPF with MPO-ANCA may possibly prevent this fatal complication and improve the prognosis of the disease although further research is imperative to confirm this hypothesis.

There are some methodological limitations in this research. First, all studies included in this review were demonstrated to contain high risk of bias in at least one domain. In particular, the study attrition was not explained clearly in all studies and study confounders and statistical analysis were also uncertain. Second, relevant autoantibodies were not necessarily tested for all patients as all included studies were of retrospective design. Accordingly, there is a possibility of selection bias of patients and diagnostic misclassification between IPF with and without autoantibodies. Third, there was substantial clinical and methodological heterogeneity between studies. An example includes diverse positivity of autoantibodies such as ANA, which may have been caused by a different cohort, different methods of testing or different cut-off values. Fourth, due to heterogeneity and a small number of included studies, meta-analysis was only possible for two different outcomes for one autoantibody. As a consequence, most of the findings obtained in this review may possibly have been affected by type 2 error in an individual study. Finally, it was difficult to draw a definitive conclusion due to the scarcity of multivariate data.

Although these potential shortcomings may prevent an immediate application of the findings of this review into daily clinical practice, this study remains meaningful in 
that it clarified current evidence regarding clinical significance of autoantibodies for IPF. Further research of highquality incorporating multivariate analysis is imperative to confirm the results of this review and to address other relevant autoantibodies and outcomes that have not been clarified in this research.

\section{CONCLUSION}

The presence of autoantibodies such as MPO-ANCA and ANA was demonstrated to be associated with the development of some autoimmune diseases for patients with IPF although there was no difference of all-cause mortality. However, the results should be interpreted with caution due to low evidence level.

Contributors HK conceived and planned the whole part of this research project. He involved the entire process of conducting this research and wrote this manuscript. $\mathrm{HK}$ will be the guarantor of the content of the review including data analysis. OMP made contributions in conceiving this research project. He also involved literature search, data extraction and risk of bias assessment. OMP added some thought and made revisions to the manuscript.

Funding The authors received no specific grant for this research from any funding agency in the public, commercial or not-for-profit sectors.

Competing interests None to declare.

Patient consent for publication Not required.

Ethics approval This systematic review and meta-analysis was based on published data. As researchers did not access any information that could lead to the identification of an individual patient, no concerning ethical issue was raised in this research. Therefore, obtaining ethical approval and consent of participants was waived.

\section{Provenance and peer review Not commissioned; externally peer reviewed.}

Data sharing statement The data set used and/or analysed for this review will be available from the corresponding author upon a reasonable request.

Open access This is an open access article distributed in accordance with the Creative Commons Attribution Non Commercial (CC BY-NC 4.0) license, which permits others to distribute, remix, adapt, build upon this work non-commercially, and license their derivative works on different terms, provided the original work is properly cited, appropriate credit is given, any changes made indicated, and the use is non-commercial. See: http://creativecommons.org/licenses/by-nc/4.0/.

\section{REFERENCES}

1. American Thoracic Society/European Respiratory Society International Multidisciplinary Consensus Classification of the Idiopathic Interstitial Pneumonias. This joint statement of the American Thoracic Society (ATS), and the European Respiratory Society (ERS) was adopted by the ATS board of directors, June 2001 and by the ERS Executive Committee, June 2001. Am J Respir Crit Care Med 2002:277-304.

2. Raghu G, Collard HR, Egan JJ, et al. An official ATS/ERS/JRS/ALAT statement: idiopathic pulmonary fibrosis: evidence-based guidelines for diagnosis and management. Am J Respir Crit Care Med 2011;183:788-824.

3. Kono M, Nakamura Y, Yoshimura K, et al. Nonspecific interstitial pneumonia preceding diagnosis of collagen vascular disease. Respir Med 2016;117:40-7.

4. Tzelepis GE, Toya SP, Moutsopoulos HM. Occult connective tissue diseases mimicking idiopathic interstitial pneumonias. Eur Respir J 2008;31:11-20.

5. Hervier B, Benveniste O. Clinical heterogeneity and outcomes of antisynthetase syndrome. Curr Rheumatol Rep 2013;15:349.

6. Mosca M, Tani C, Bombardieri S. Undifferentiated connective tissue diseases (UCTD): a new frontier for rheumatology. Best Pract Res Clin Rheumatol 2007;21:1011-23.

7. Kinder BW, Collard HR, Koth L, et al. Idiopathic nonspecific interstitial pneumonia: lung manifestation of undifferentiated connective tissue disease? Am J Respir Crit Care Med 2007;176:691-7.

8. Fischer A, West SG, Swigris JJ, et al. Connective tissue diseaseassociated interstitial lung disease: a call for clarification. Chest 2010;138:251-6.

9. Vij R, Noth I, Strek ME. Autoimmune-featured interstitial lung disease: a distinct entity. Chest 2011;140:1292-9.

10. Fischer A, Antoniou KM, Brown KK, et al. An official European Respiratory Society/American Thoracic Society research statement: interstitial pneumonia with autoimmune features. Eur Respir $J$ 2015;46:976-87.

11. Kim EJ, Elicker BM, Maldonado F, et al. Usual interstitial pneumonia in rheumatoid arthritis-associated interstitial lung disease. Eur Respir $J$ 2010;35:1322-8.

12. Kim EA, Lee KS, Johkoh $\mathrm{T}$, et al. Interstitial lung diseases associated with collagen vascular diseases: radiologic and histopathologic findings. Radiographics 2002;22 Spec No:S151-65.

13. Lee JS, Kim EJ, Lynch KL, et al. Prevalence and clinical significance of circulating autoantibodies in idiopathic pulmonary fibrosis. Respir Med 2013;107:249-55.

14. Centre for Reviews and Dissemination, University of York. PROSPERO: international prospective register of systematic reviews. http://www.crd.york.ac.uk/prospero/ (Accessed 30 Sep 2018).

15. Moher D, Liberati A, Tetzlaff J, et al. Preferred reporting items for systematic reviews and meta-analyses: the PRISMA statement. Ann Intern Med 2009;151:264-9.

16. Stroup DF, Berlin JA, Morton SC, et al. Meta-analysis of observational studies in epidemiology: a proposal for reporting. Meta-analysis Of Observational Studies in Epidemiology (MOOSE) group. JAMA 2000;283:2008-12.

17. Kamiya $\mathrm{H}$, Panlaqui OM. Prognostic significance of autoantibodies for idiopathic pulmonary fibrosis: protocol for a systematic review. BMJ Open 2018;8:e020862.

18. Foulon G, Delaval P, Valeyre D, et al. ANCA-associated lung fibrosis: analysis of 17 patients. Respir Med 2008;102:1392-8.

19. Wilczynski NL, Haynes RB, Hedges Team. Developing optimal search strategies for detecting clinically sound prognostic studies in MEDLINE: an analytic survey. BMC Med 2004;2:23.

20. Wilczynski NL, Haynes RB. Optimal search strategies for detecting clinically sound prognostic studies in EMBASE: an analytic survey. $J$ Am Med Inform Assoc 2005;12:481-5.

21. Haddaway NR, Collins AM, Coughlin D, et al. The role of google scholar in evidence reviews and its applicability to grey literature searching. PLoS One 2015;10:e0138237.

22. Hayden JA, Côté P, Bombardier C. Evaluation of the quality of prognosis studies in systematic reviews. Ann Intern Med 2006;144:427-37.

23. Tierney JF, Stewart LA, Ghersi D, et al. Practical methods for incorporating summary time-to-event data into meta-analysis. Trials 2007;8:16.

24. DerSimonian R, Laird N. Meta-analysis in clinical trials. Control Clin Trials 1986;7:177-88.

25. Riley RD, Higgins JP, Deeks JJ. Interpretation of random effects meta-analyses. BMJ 2011;342:d549.

26. Higgins JPT, Green S. Cochrane handbook for systematic reviews of interventions version 5.1.0 [updated march 2011]. the cochrane collaboration. $2011 \mathrm{http}: / / \mathrm{www}$. handbook.cochrane.org.

27. Egger M, Davey Smith G, Schneider M, et al. Bias in meta-analysis detected by a simple, graphical test. BMJ 1997;315:629-34.

28. Iorio A, Spencer FA, Falavigna M, et al. Use of GRADE for assessment of evidence about prognosis: rating confidence in estimates of event rates in broad categories of patients. BMJ 2015;350:h870.

29. Kang BH, Park JK, Roh JH, et al. Clinical significance of serum autoantibodies in idiopathic interstitial pneumonia. J Korean Med Sci 2013;28:731-7.

30. Moua T, Maldonado F, Decker PA, et al. Frequency and implication of autoimmune serologies in idiopathic pulmonary fibrosis. Mayo Clin Proc 2014;89:319-26.

31. Song JW, Do KH, Kim MY, et al. Pathologic and radiologic differences between idiopathic and collagen vascular disease-related usual interstitial pneumonia. Chest 2009;136:23-30.

32. Kagiyama N, Takayanagi N, Kanauchi T, et al. Antineutrophil cytoplasmic antibody-positive conversion and microscopic polyangiitis development in patients with idiopathic pulmonary fibrosis. BMJ Open Respir Res 2015;2:e000058.

33. Hosoda C, Baba T, Hagiwara E, et al. Clinical features of usual interstitial pneumonia with anti-neutrophil cytoplasmic antibody in comparison with idiopathic pulmonary fibrosis. Respirology 2016;21:920-6. 
34. Nozu T, Kondo M, Suzuki K, et al. A comparison of the clinical features of ANCA-positive and ANCA-negative idiopathic pulmonary fibrosis patients. Respiration 2009;77:407-15.

35. Hozumi H, Enomoto N, Oyama $\mathrm{Y}$, et al. Clinical Implication of Proteinase-3-antineutrophil Cytoplasmic Antibody in Patients with Idiopathic Interstitial Pneumonias. Lung 2016;194:235-42.

36. Hozumi H, Oyama Y, Yasui H, et al. Clinical significance of myeloperoxidase-anti-neutrophil cytoplasmic antibody in idiopathic interstitial pneumonias. PLoS One 2018;13:e0199659.

37. Wolters PJ, Collard HR, Jones KD. Pathogenesis of idiopathic pulmonary fibrosis. Annu Rev Pathol 2014;9:157-79.

38. Li FJ, Surolia R, Li H, et al. Autoimmunity to vimentin is associated with outcomes of patients with idiopathic pulmonary fibrosis. $J$ Immunol 2017;199:1596-605.

39. Feghali-Bostwick CA, Tsai CG, Valentine VG, et al. Cellular and humoral autoreactivity in idiopathic pulmonary fibrosis. $\mathrm{J}$ Immunol 2007;179:2592-9.

40. Feghali-Bostwick CA, Wilkes DS. Autoimmunity in idiopathic pulmonary fibrosis: are circulating autoantibodies pathogenic or epiphenomena? Am J Respir Crit Care Med 2011;183:692-3.

41. Solomon DH, Kavanaugh AJ, Schur PH. Evidence-based guidelines for the use of immunologic tests: antinuclear antibody testing. Arthritis Rheum 2002;47:434-44.

42. Shmerling RH, Delbanco TL. The rheumatoid factor: an analysis of clinical utility. Am J Med 1991;91:528-34.

43. Bahmer T, Romagnoli M, Girelli F, et al. The use of auto-antibody testing in the evaluation of interstitial lung disease (ILD)--A practical approach for the pulmonologist. Respir Med 2016;113:80-92.

44. Kelly CA, Saravanan V, Nisar M, et al. Rheumatoid arthritis-related interstitial lung disease: associations, prognostic factors and physiological and radiological characteristics--a large multicentre UK study. Rheumatology 2014;53:1676-82.

45. Kallenberg CG. Pathogenesis of ANCA-associated vasculitides. Ann Rheum Dis 2011;70(Suppl 1):i59-i63.

46. Kono M, Nakamura $\mathrm{Y}$, Enomoto $\mathrm{N}$, et al. Usual interstitial pneumonia preceding collagen vascular disease: a retrospective case control study of patients initially diagnosed with idiopathic pulmonary fibrosis. PLoS One 2014;9:e94775117.

47. Alhamad EH, Al-Kassimi FA, Alboukai AA, et al. Comparison of three groups of patients with usual interstitial pneumonia. Respir Med 2012;106:1575-85

48. Riha RL, Duhig EE, Clarke BE, et al. Survival of patients with biopsyproven usual interstitial pneumonia and nonspecific interstitial pneumonia. Eur Respir J 2002;19:1114-8.

49. Nunes H, Schubel K, Piver D, et al. Nonspecific interstitial pneumonia: survival is influenced by the underlying cause. Eur Respir J 2015;45:746-55.

50. Park JH, Kim DS, Park IN, et al. Prognosis of fibrotic interstitial pneumonia: idiopathic versus collagen vascular disease-related subtypes. Am J Respir Crit Care Med 2007;175:705-11.

51. Ando M, Miyazaki E, Ishii T, et al. Incidence of myeloperoxidase anti-neutrophil cytoplasmic antibody positivity and microscopic polyangitis in the course of idiopathic pulmonary fibrosis. Respir Med 2013;107:608-15.

52. Kallenberg CG. Pathogenesis of PR3-ANCA associated vasculitis. $J$ Autoimmun 2008:30:29-36.

53. Homma S, Suzuki A, Sato K. Pulmonary involvement in ANCAassociated vasculitis from the view of the pulmonologist. Clin Exp Nephrol 2013;17:667-71. 